\title{
Confined rotating convection with large Prandtl number: Centrifugal effects on wall modes
}

\author{
Jezabel Curbelo \\ Instituto de Ciencias Matemáticas (CSIC-UAM-UC3M-UCM), Nicolás Cabrera, 15, Madrid 28049, Spain \\ and Departamento de Matemáticas, Universidad Autónoma de Madrid, Madrid 28049, Spain \\ Juan M. Lopez ${ }^{*}$ \\ School of Mathematical and Statistical Sciences, Arizona State University, Tempe, Arizona 85287, USA \\ Ana M. Mancho \\ Instituto de Ciencias Matemáticas (CSIC-UAM-UC3M-UCM), Nicolás Cabrera, 15, Madrid 28049, Spain \\ Francisco Marques \\ Department of Física Aplicada, Universitat Politècnica de Catalanya, Girona s/n, Mòdul B4 Campus Nord, 08034 Barcelona, Spain
}

(Received 29 October 2013; published 23 January 2014)

\begin{abstract}
Thermal convection in a rotating cylinder with a radius-to-height aspect ratio of $\Gamma=4$ for fluids with large Prandtl number is studied numerically. Centrifugal buoyancy effects are investigated in a regime where the Coriolis force is relatively large and the onset of thermal convection is in the so-called wall modes regime, where pairs of hot and cold thermal plumes ascend and descend in the cylinder sidewall boundary layer, forming an essentially one-dimensional pattern characterized by the number of hot and cold plume pairs. In our numerical study, we use the physical parameters corresponding to aqueous mixtures of glycerine with mass concentration in the range of 60\%-90\% glycerine and a Rayleigh number range that extends from the threshold for wall modes up to values where the bulk fluid region is also convecting. The study shows that for the range of Rayleigh numbers considered, the local variations in viscosity due to temperature variation in the flow are negligible. However, the mean viscosity, which varies faster than exponentially with variations in the percentage of glycerine, leads to a faster than exponential increase in the Froude number for a fixed Coriolis force, and hence an enhancement of the centrifugal buoyancy effects with significant dynamical consequences, which are detailed.
\end{abstract}

DOI: 10.1103/PhysRevE.89.013019

PACS number(s): 47.32.Ef, 47.55.pb

\section{INTRODUCTION}

Rotating Rayleigh-Bénard convection in a circular cylinder is a classical canonical system that has been receiving much additional attention recently due to the availability of new modern experimental facilities and significant advances in its numerical simulation [1]. For the most part, studies have focused on Prandtl numbers (the ratio of thermal to viscous time scales of the problem) of order 10 and smaller, motivated primarily by astrophysical interests [2]. Large Prandtl number systems are also of much interest, particularly when the working fluids are various alcohols, silicone oils, and exotic gases under high pressure. For this reason it is desirable to access high-Prandtl number regimes both in laboratory experiments and in numerical or theoretical models in order to gain insight into some of the physical processes involved. Conducting laboratory experiments over a range of large Prandtl numbers requires the choice of appropriate fluids. Aqueous mixtures of glycerine are one such choice which has been used in many fluid mechanical settings [3], as well as in heat-transfer and chemical kinetics applications. However, these have a temperature-dependent viscosity which may need to be accounted for.

In rotating convection, recent experiments [4] have reported significant qualitative differences between the states found for Prandtl numbers bigger than one and smaller than one. In

*jmlopez@asu.edu
Ref. [5] heat flux enhancements due to rotation were investigated, and it was noted that there is a lack of experimental and numerical studies that address Prandtl number effects for rotating convection. For Prandtl numbers greater than 100, there are few studies in rotating convection. There exist some theoretical studies in the limit of infinite Prandtl number [6-8], but these neglect two aspects of rotating convection which can be dominant, especially in a realistic physical setting, namely, confinement and centrifugal buoyancy [9-14]. Experiments are often designed to operate in parameter regimes where the Froude number Fr is small so that centrifugal effects can be neglected [15]. Neglecting centrifugal buoyancy in a large Prandtl number setting can be especially problematic since reaching a large Prandtl number requires a large viscosity fluid, such as highly concentrated aqueous mixtures of glycerine. As the Coriolis number (ratio of viscous time to system rotation period) also depends on the viscosity, in order to keep this number fixed while increasing the Prandtl number, the rotation of the system must be increased. This increment also augments the Froude number, which depends, quadratically, on the rotation rate. So, for small Prandtl numbers the Froude number is small and generally negligible, but for large Prandtl numbers the Froude number becomes large enough to strongly influence the dynamics.

In this paper, we conduct numerical simulations in a rotating cylinder of radius-to-depth ratio equal to 4 with fluids covering a large range of Prandtl and Froude numbers. The precise parameter regimes are described below, together with the description of the governing equations and their numerical 
resolution. We find that for moderate Rayleigh numbers (up to and a little beyond where the bulk begins to convect), the local variations in viscosity due to temperature variations in the flow are negligible, especially when compared to the centrifugal effects.

\section{THE PHYSICAL SETUP, THE GOVERNING EQUATIONS, AND THE NUMERICAL SCHEME}

Consider the flow in a circular cylinder of radius $r_{0}$ and depth $d$, rotating at a constant rate $\omega \mathrm{rad} / \mathrm{s}$. The top and bottom end walls are maintained at constant temperatures $T_{0}-0.5 \Delta T$ and $T_{0}+0.5 \Delta T$, respectively, where $T_{0}$ is the reference temperature and $\Delta T$ is the temperature difference between the end walls. The sidewall is assumed to be insulating. The governing equations are written in the rotating frame of reference, using the Boussinesq approximation in which all fluid properties are considered constant, except for the density in the gravitational and centrifugal buoyancy terms. Using $d$ as the length scale, $d^{2} / \kappa$ as the time scale, $v^{2} \rho_{0} / d^{2}$ as the pressure scale ( $\rho_{0}$ is the density at temperature $T_{0}$ ), and $\Delta T$ as the temperature scale, the nondimensional governing equations are

$$
\begin{gathered}
\left(\partial_{t}+\boldsymbol{u} \cdot \nabla\right) \boldsymbol{u}=-\nabla p+\sigma \operatorname{Ra} T \hat{z}+2 \sigma \Omega \boldsymbol{u} \times \hat{z} \\
-\frac{\sigma \operatorname{FrRa}}{\Gamma} T \boldsymbol{r}+\sigma \nabla^{2} \boldsymbol{u}, \\
\left(\partial_{t}+\boldsymbol{u} \cdot \nabla\right) T=\nabla^{2} T, \quad \nabla \cdot \boldsymbol{u}=0,
\end{gathered}
$$

where the nondimensional temperature is $T=\left(T_{\text {phys }}-\right.$ $\left.T_{0}\right) / \Delta T, \boldsymbol{u}$ is the velocity field in the rotating frame, $(u, v, w)$ are the components of $\boldsymbol{u}$ in cylindrical coordinates $(r, \theta, z), p$ is the kinematic pressure (including gravitational and centrifugal contributions), $\hat{z}$ the unit vector in the vertical direction $z$, and $\boldsymbol{r}$ is the radial vector in cylindrical coordinates. There are five nondimensional parameters:

$$
\begin{aligned}
& \text { Rayleigh number } \\
& \mathrm{Ra}=\alpha g d^{3} \Delta T / \kappa \nu_{0} \\
& \text { Coriolis number } \\
& \Omega=\omega d^{2} / \nu_{0} \\
& \text { Froude number } \\
& \mathrm{Fr}=\omega^{2} r_{0} / g \\
& \text { Prandtl number } \\
& \sigma=v_{0} / \kappa \\
& \text { Aspect ratio } \\
& \Gamma=r_{0} / d,
\end{aligned}
$$

(a)

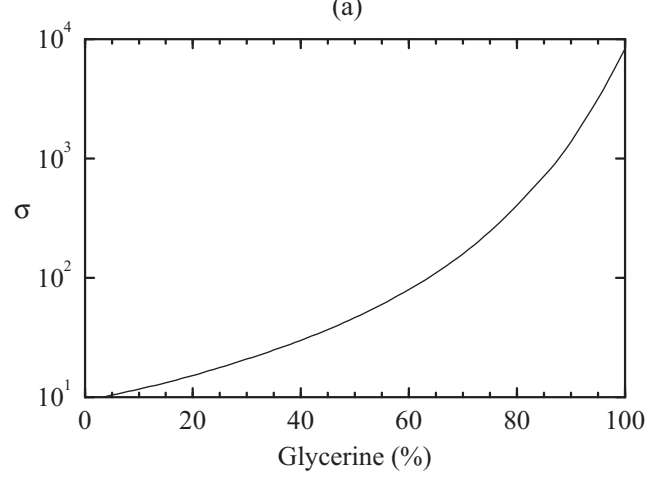

where $\alpha$ is the coefficient of volume expansion, $g$ is the gravitational acceleration, $\kappa$ is the thermal diffusivity, and $v_{0}$ is the kinematic viscosity at temperature $T_{0}$. The boundary conditions for $\boldsymbol{u}$ and $T$ are

$$
\begin{array}{rll}
r=\Gamma: & u=v=w=0, & T_{r}=0, \\
z= \pm 1 / 2: & u=v=w=0, & T=\mp 1 / 2 .
\end{array}
$$

The dimensionless form of the temperature-dependent density used in (1) is

$$
\rho(T)=\rho_{0}(1-\alpha T) .
$$

The validity of the Boussinesq approximation requires $\alpha \Delta T \ll 1$. Expressing this value in terms of the nondimensional parameters of the problem gives

$$
\alpha \Delta T=\operatorname{RaFr} / \Omega^{2} \sigma \Gamma .
$$

For the range of parameters considered in this study, $\alpha \Delta T$ is indeed small $\left(<10^{-3}\right)$. This criterion is often quoted as being a sufficient criterion to validate the Boussinesq approximation, although Refs. [16,17] introduced additional conditions under which the Boussinesq approximation can be applied. These include certain factors related to the temperature dependence of the material properties, which should be smaller than a certain tolerance for a required level of accuracy. For the parameter regimes considered in this study, since $\Delta T$ is small, these requirements are also satisfied.

In our numerical experiments, we have considered glycerine-water mixtures. The aspect ratio is fixed to $\Gamma=4$ and the Coriolis number to $\Omega=625$, corresponding to values used in previous studies where onset was dominated by wall modes [18,19]. Figure 1 shows the variations of $\sigma$ and $\mathrm{Fr}$ for aqueous mixtures of glycerine at $22.4{ }^{\circ} \mathrm{C}$. The density and dynamic viscosity of the aqueous glycerine mixtures, required to calculate $\sigma$ and $\mathrm{Fr}$, have been obtained from Ref. [20]. We have focused our attention on the concentration range $60 \%-90 \%$, as we are interested in examining centrifugal effects and for glycerine concentrations less than $60 \%$, Fr $<$ 0.01 , and the effects of this parameter are negligible. For the range of glycerine concentrations considered, the Prandtl number increases by about one order of magnitude while the Froude number increases by almost two orders of magnitude. Temperature-dependent viscosity effects are negligible in the parameter range analyzed in this study. For completeness,

(b)

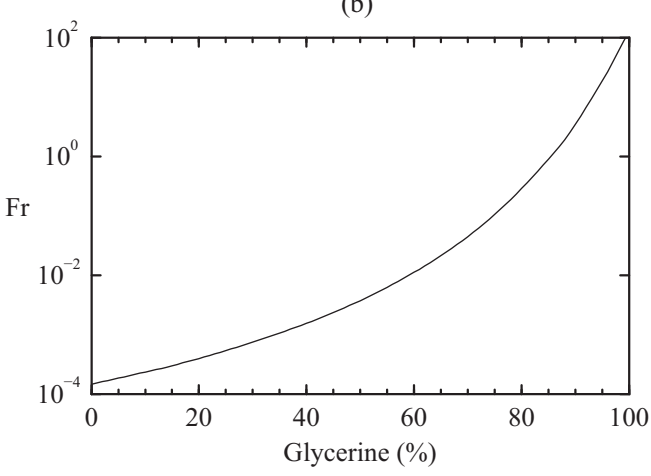

FIG. 1. Variations of $\sigma$ and $\mathrm{Fr}$ for aqueous mixtures of glycerine at $22.4{ }^{\circ} \mathrm{C}$. 
these are briefly discussed in the Appendix, which also details how the governing equations change due to temperaturedependent viscosity, and how these are solved numerically.

The governing equations have been solved using the second-order time-splitting method proposed in Ref. [21] combined with a pseudospectral method for the spatial discretization, utilizing a Galerkin-Fourier expansion in the azimuthal coordinate $\theta$ and Chebyshev collocation in $x=r / \Gamma$ and $y=2 z$ of the form

$$
F(r, \theta, z)=\sum_{l=0}^{L} \sum_{n=0}^{N} \sum_{m=-M}^{M} a_{l, n, m} \Xi_{l}(x) \Xi_{n}(y) e^{\mathrm{i} m \theta} .
$$

The velocity components, temperature, and pressure are the real or imaginary parts of $F$. The radial dependence of the variables is approximated by Chebyshev expansions with appropriate parities of their azimuthal Fourier components [22]. To avoid including the origin in the collocation mesh, an odd number of Gauss-Lobatto points in $r$ is used and the equations are solved only in the interval $r \in(0, \Gamma]$. Following Ref. [23], we have used the combinations $u_{+}=u+i v$ and $u_{-}=u-i v$ in order to decouple the linear diffusion terms in the momentum equations. For each Fourier mode, the resulting Helmholtz equations for $T, w, u_{+}$, and $u_{-}$have been solved using a diagonalization technique in the two coordinates $r$ and $z$. The imposed parity of the Fourier modes guarantees the regularity conditions at the origin needed to solve the Helmholtz equations [24]. Further details of the numerical code can be found in Ref. [25]. We have used $L=48$ spectral modes in $r, N=24$ in $z, M=184$ in $\theta$, and a time step $d t \in[2.5 \times$ $\left.10^{-7}, 5 \times 10^{-6}\right]$ thermal time units. The time step used depends on the Prandtl number and to a lesser extent the Froude number. When these are large, the time step used needs to be decreased.

\section{RESULTS}

The present study is focused on examining how the wall modes in rotating convection are quenched in a regime dominated by Coriolis force and large Prandtl number. For a fixed Coriolis number, we consider a variety of aqueous mixtures of glycerine, thus setting the Froude and Prandtl numbers of each mixture, and increase the Rayleigh number from below the onset of the wall modes to beyond values at which bulk convection occurs.

Our results can be characterized by the Nusselt number $\mathrm{Nu}$, the ratio of the vertical heat flux of the flow state to the vertical heat flux due solely to conduction:

$$
\mathrm{Nu}(r, \theta, z)=-\partial_{z} T(r, \theta, z) .
$$

It is also useful to consider the azimuthally averaged Nusselt number given by

$$
\overline{\mathrm{Nu}}(r, z)=-\int_{0}^{2 \pi} \partial_{z} T(r, \theta, z) d \theta,
$$

and the horizontally averaged Nusselt number $\langle\mathrm{Nu}\rangle$ at any height $z$, given by

$$
\begin{aligned}
\langle\mathrm{Nu}\rangle(z) & =-\int_{0}^{\Gamma} \int_{0}^{2 \pi} \partial_{z} T(r, \theta, z) r d r d \theta \\
& =\int_{0}^{\Gamma} \overline{\mathrm{Nu}}(r, z) r d r .
\end{aligned}
$$

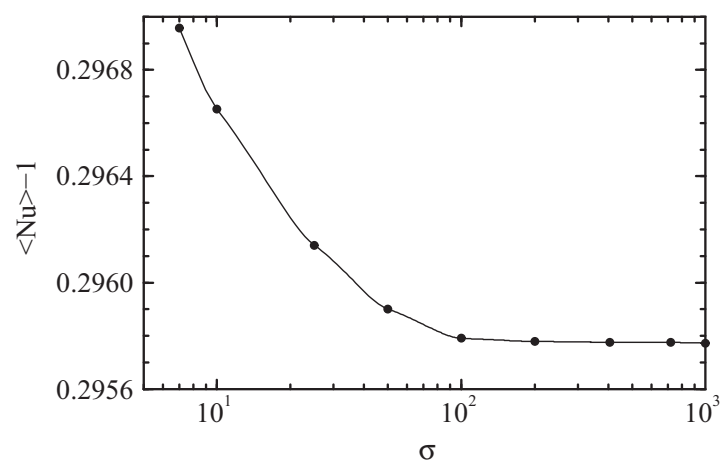

FIG. 2. Variation of the Nusselt number with $\sigma$ for $\Omega=625$, $\mathrm{Ra}=9 \times 10^{4}, \Gamma=4$, and $\mathrm{Fr}=0$.

We begin by examining the effects of increasing the Prandtl number while ignoring the centrifugal buoyancy (artificially setting $\mathrm{Fr}=0$ ), as this corresponds to a setting closer to previous studies on Prandtl number effects and provides a case to compare with when centrifugal buoyancy is accounted for.

We set $\mathrm{Ra}=9 \times 10^{4}$, which is well above the critical Rayleigh number for the onset of wall modes, $\operatorname{Ra}_{c} \sim 4 \times 10^{4}$, when $\Omega=625$ and $\Gamma=4$. In this parameter regime, the wall modes are stable and there is no bulk convection. The wall modes are rotating waves whose spatial structure simply precesses without change (in the rotating frame of reference), thus leading to a time-independent Nusselt number [26]. This Rayleigh number is close to but below the value for which bulk convection occurs. The wall mode structure is maintained for all values of $\sigma$ without any significant differences for solutions with $\sigma>100$.

Figure 2 shows how the Nusselt number varies with $\sigma \in[7,1000]$. The Nusselt number diminishes slightly with increasing $\sigma$ until $\sigma \sim 100$, and from then on it is independent of $\sigma$. The temperature and velocity throughout the cylinder do not vary with Prandtl number for $\sigma>100$. This is consistent with what is found generally in nonrotating convection, but as we shall now see, is a result of ignoring centrifugal buoyancy effects.

Typically, in rotating convection studies, the Coriolis term is taken into account but the Froude number is artificially set to zero, ignoring the centrifugal buoyancy term. Refs. [12,27] have shown that centrifugal buoyancy plays a significant dynamic role and changes the problem in a fundamental manner. The total buoyancy force (gravitational plus centrifugal) no longer points in the vertical direction, but now also has a radial component which varies with radial distance from the rotation axis. This destroys the horizontal translation invariance that is inherent in the horizontally unbounded theoretical treatments of the problem [7,28-32]. Furthermore, the reflection symmetry about the cylinder midheight (the socalled up-down symmetry) is also destroyed. Figure 1 strongly suggests that for the fixed Coriolis number $\Omega=625$, one cannot ignore centrifugal buoyancy because for the glycerine mixtures considered, the corresponding Froude number is not small $(0.01 \lesssim$ Fr $\lesssim 10)$.

For $\mathrm{Ra} \in\left[2 \times 10^{4}, 9 \times 10^{4}\right]$, we now study the effects of $\mathrm{Fr} \neq 0$. We do this by increasing the mass concentration 


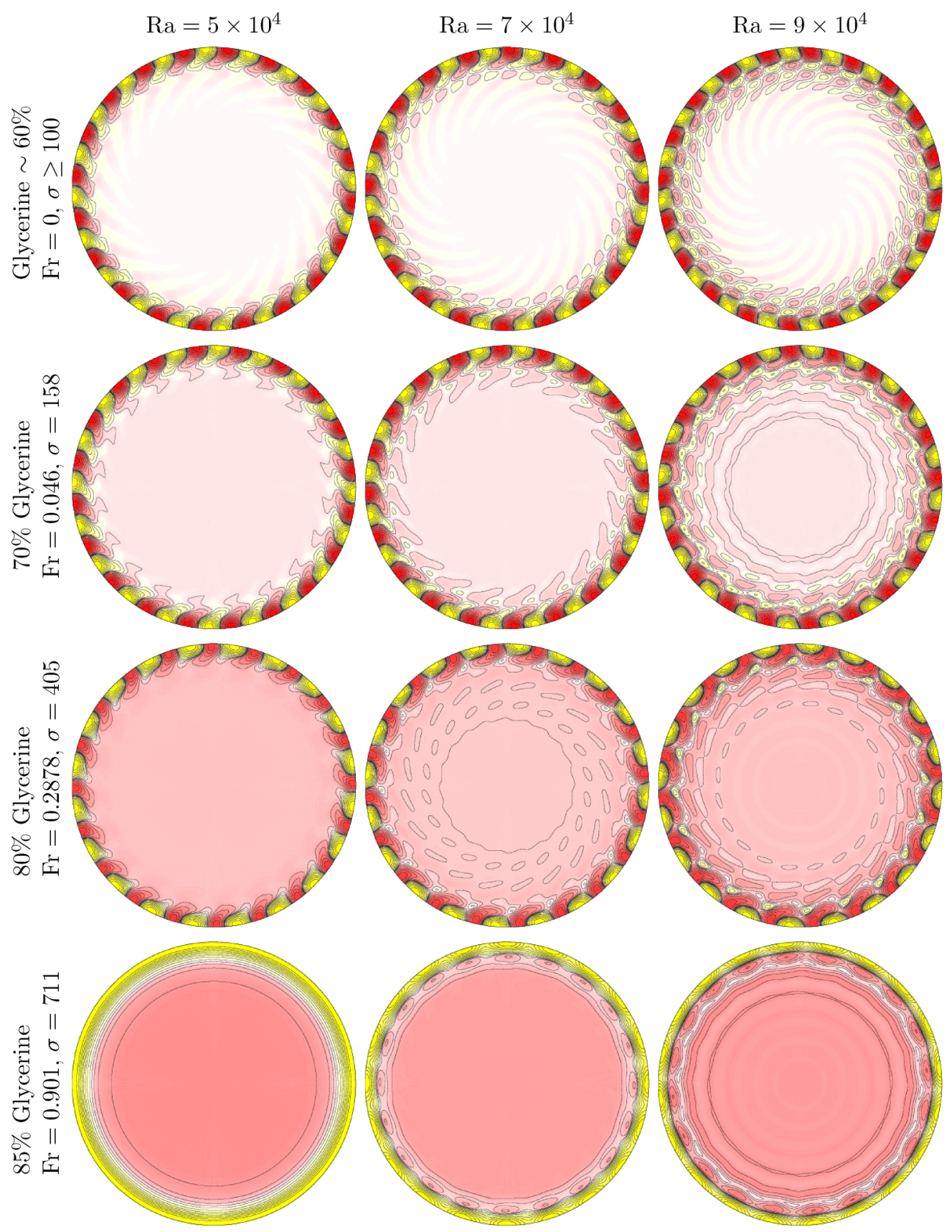

FIG. 3. (Color online) Isotherms at height $z=0.2$ for $\Omega=625, \Gamma=4$, and Ra as indicated, for $\sigma=100$ ignoring centrifugal buoyancy (top row with $\mathrm{Fr}=0$ ), and with the values of $\mathrm{Fr}$ and $\sigma$ corresponding to aqueous mixtures of glycerine at $70 \%$ (second row), $80 \%$ (third row), and $85 \%$ (bottom row) of mass at $22.4{ }^{\circ} \mathrm{C}$. There are 10 positive (red/dark gray) and 10 negative (yellow/light gray) isotherms in the range $T \in[-0.5,0.5]$.

of glycerine in aqueous mixtures, which also increases the viscosity of the fluid. In order to keep the Coriolis number the same for all aqueous mixtures, the rotation rate of the cylinder $\omega$ must increase with the viscosity, i.e., the concentration of glycerine, and hence the Froude number Fr also increases. We have considered several glycerine concentrations. For glycerine concentration of about $60 \%$, Fr $\sim 10^{-2}$ is small and negligible and $\sigma \sim 100$, and so we set the test case with which to compare the results for larger glycerine concentrations to be $\mathrm{Fr}=0$ and $\sigma=100$, nominally corresponding to a $60 \%$ glycerine mixture. Figure 3 shows isotherms in a horizontal section of the cylinder at $z=0.2$ for $\Omega=625$ and $\Gamma=4$, for four representative cases at $\mathrm{Ra}$ as indicated. In the first row, we have the nominal cases for $60 \%$ glycerine mixture with artificially set $\mathrm{Fr}=0$ and $\sigma=100$. In fact, with $\mathrm{Fr}=0$, there are no discernable changes in the solutions for $\sigma>100$ over the range of $\mathrm{Ra}$ considered in this paper. The other three rows in the figure are for increasing percentages of glycerine in the mixture and are computed with the corresponding physical values of Fr and $\sigma$, as indicated. In all simulations, we begin with the lowest value of $\mathrm{Ra}$ and use as initial condition the wall mode with azimuthal wave number $m=20$ computed 


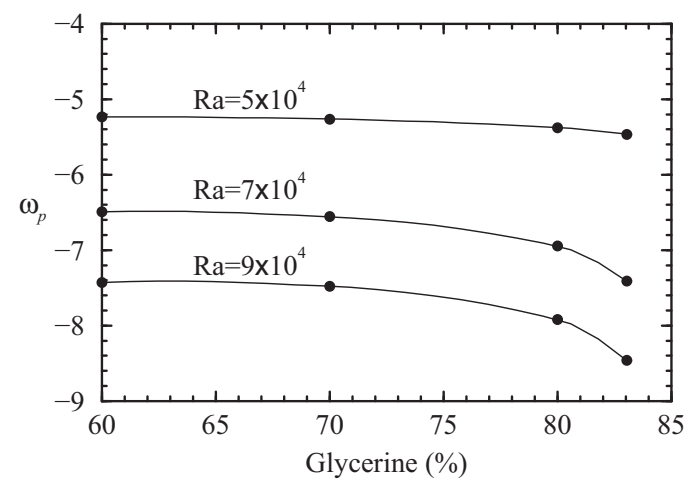

FIG. 4. Variation of the precession frequency of the wall modes with glycerine concentration for various $\operatorname{Ra}$ with $\Omega=625$ and $\Gamma=4$; the values of $\sigma$ and Fr used are for the corresponding glycerine mixture at $22.4^{\circ} \mathrm{C}$.

for $\mathrm{Fr}=0, \sigma=7, \Gamma=4$, and $\Omega=625$. In this parameter regime there is a large Eckhaus-stable band of wall modes with azimuthal wave numbers varying from about 12 to $36[18,19]$. By using the same initial condition for low $\mathrm{Ra}$ and continuing the resulting wall mode state to higher $\mathrm{Ra}$, we are able to continue the same wall mode branch for the different glycerine concentrations.

The wall-mode structure is maintained for small values of the Froude number; it becomes weaker at intermediate Fr and is quenched for large Fr, where axisymmetric target-like patterns are found. As Fr increases, the precession frequency also changes, as shown in Fig. 4. The precession of the wall modes is retrograde in all cases considered and increases in absolute value when Fr increases, and the increase is larger for increasing $\mathrm{Ra}$. As the percentage of glycerine is reduced, the precession frequencies $\omega_{p}$ approach those reported in Ref. [19], which considered the case of pure water with $\mathrm{Fr}=0$ and $\sigma=7$. The precession frequencies $\omega_{p}$ reported here and in Ref. [19] correspond to the frequencies obtained via FFT of the temperature at a fixed point; the rotation frequency of

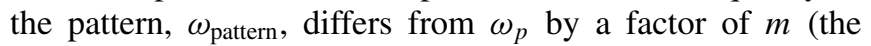
azimuthal wave number $m=20$ in the case considered here): $\omega_{\text {pattern }}=\omega_{p} / m$.

Figure 5(a) shows how $\langle\mathrm{Nu}\rangle-1$, computed at the top end wall $z=0.5$, varies with the glycerine concentration for various $\mathrm{Ra} \leqslant 9 \times 10^{4}$, for which the Nusselt number remains time independent. Figures 5(b) and 5(c) show the same result but as functions of the corresponding values of Fr and $\sigma$. The minima in the curves correspond to the glycerine concentrations (and Fr and $\sigma$ ) at which the wall modes are quenched for the given $\mathrm{Ra}$. To the left of these minima (light gray shaded area), wall modes are present, and to the right (dark gray shaded area) the wall modes are not present and the centrifugal buoyancy driven target patterns are observed. For low glycerine concentrations, Fr is small, and as the concentration is increased the centrifugal buoyancy strength increases, acting most strongly at large radii where the wall mode plumes reside; it acts to diminish the strength of the wall modes so that they transport less heat. At the same time, the centrifugal buoyancy is also driving an axisymmetric large-scale meridional circulation that is enhancing the heat
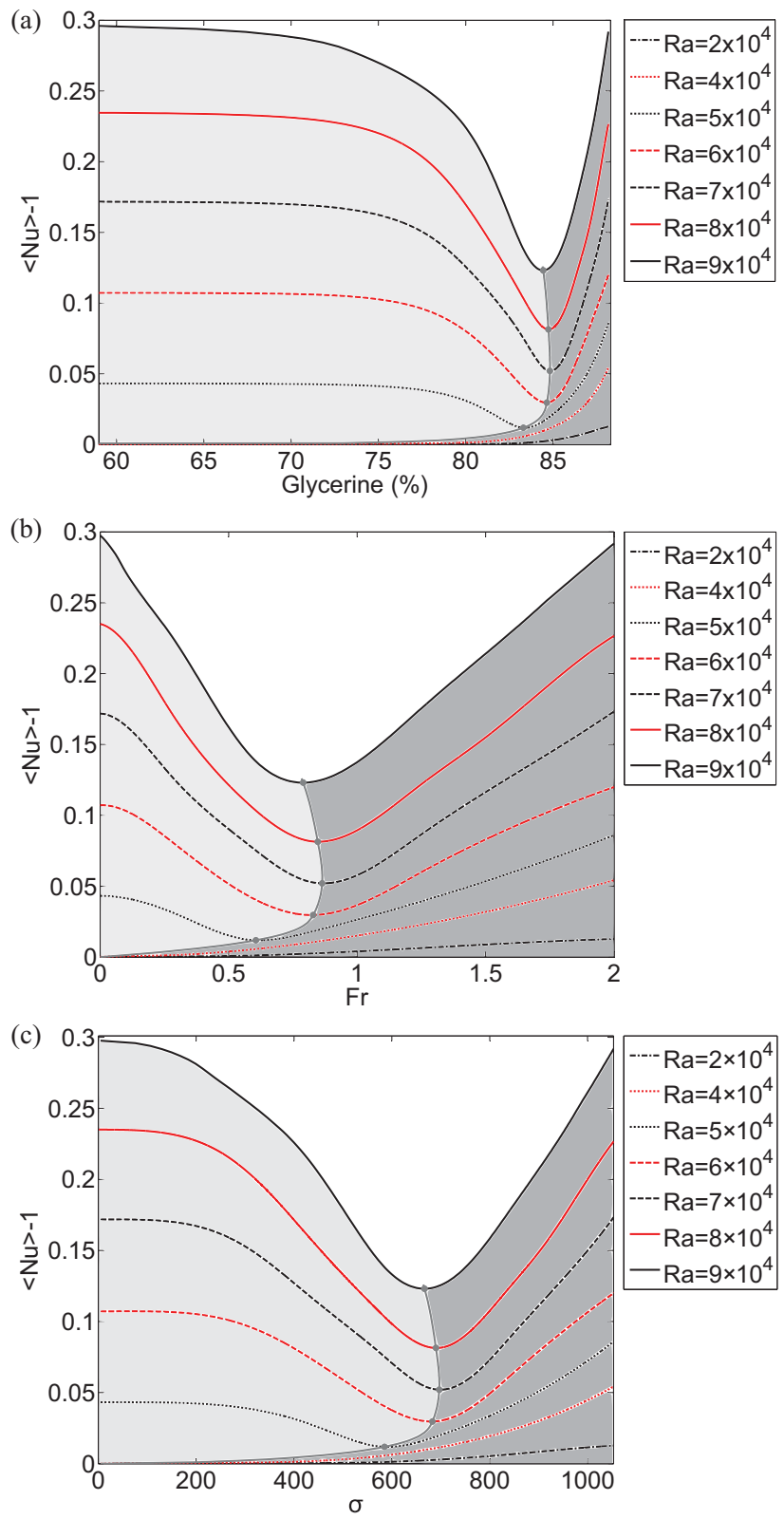

FIG. 5. (Color online) Nusselt number versus (a) glycerine concentration, (b) Froude number, and (c) Prandtl number for $\Omega=625$ and $\Gamma=4$ and various $\mathrm{Ra}$ as indicated. The values of $\sigma$ and Fr used are for the corresponding glycerine mixture at $22.4{ }^{\circ} \mathrm{C}$.

transport and is responsible for the rapid increase in the Nusselt number at large glycerine concentrations where Fr is also large. Above $\mathrm{Ra}=9 \times 10^{4}$, the onset of bulk convection is observed.

Figure 6 is the continuation of Fig. 3 to higher Rayleigh numbers. As expected, for fixed glycerine concentration (or fixed $\mathrm{Fr}$ and $\sigma$ ), as the Rayleigh number is increased the bulk interior fluid starts to convect. On the other hand, for $\mathrm{Ra}$ fixed, an increase in the glycerine concentration enhances the centrifugal buoyancy, and not only are the wall modes quenched but so too is the bulk convection. When there is bulk convection at low glycerine concentrations, the time evolution of the patterns is of Küppers-Lortz type [33], with rolls changing their orientation in time (see Refs. [13,19] 

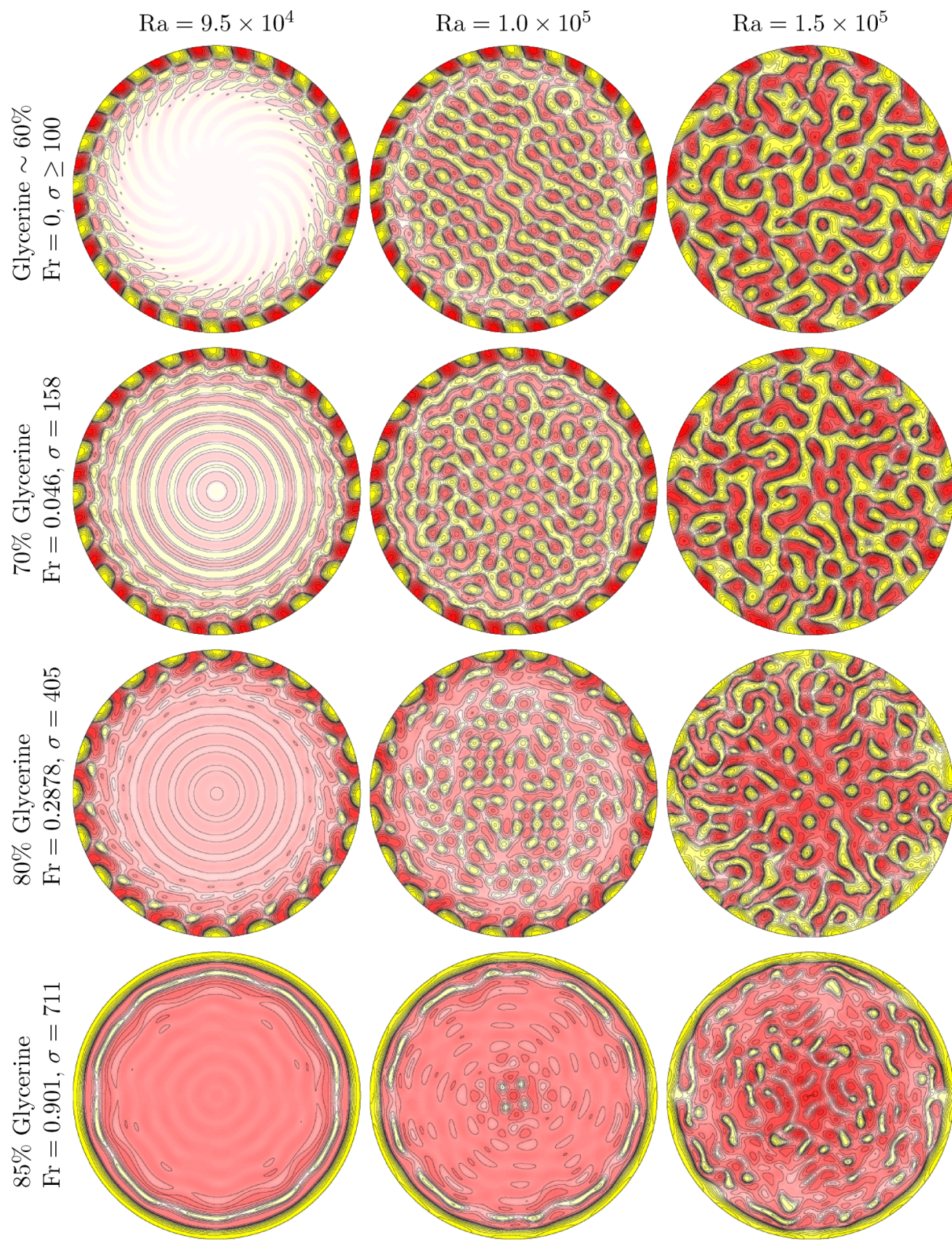

FIG. 6. (Color online) Isotherms at height $z=0.2$ for $\Omega=625, \Gamma=4$, and Ra as indicated, for $\sigma=100$ ignoring centrifugal buoyancy (top row with $\mathrm{Fr}=0$ ), and with the values of $\mathrm{Fr}$ and $\sigma$ corresponding to aqueous mixtures of glycerine at $70 \%$ (second row), $80 \%$ (third row), and $85 \%$ (bottom row) of mass at $22.4{ }^{\circ} \mathrm{C}$. There are 10 positive (red/dark gray) and 10 negative (yellow/light gray) isotherms in the range $T \in[-0.5,0.5]$.

for similar simulations with $\mathrm{Fr}=0$ ). However, this dynamic is destroyed for high glycerine concentrations as centrifugal buoyancy effects become more dominant.

Figure 7 depicts the azimuthally averaged Nusselt number $\overline{\mathrm{Nu}}(r, z)$ defined in (10), versus the radial distance $r$ at the upper plate $(z=0.5)$. We observe that most of the heat flux occurs in the sidewall boundary layer where either the wall mode plumes reside when Fr is small, or the target pattern rings have the strongest circulation when Fr is large. The Nusselt number increases with the Rayleigh number, but is always localized in the sidewall boundary layer until $\mathrm{Ra}$ is increased above the onset of bulk convection. In the highest glycerine concentration case with $\mathrm{Fr}=0.901$, for $\mathrm{Ra}=10^{5}$, just as the bulk is about to convect, we see that there is a strong correlation between the heat flux radial distribution and the strength of the circulation in the target pattern rings.

\section{CONCLUSIONS}

This paper addresses the study of rotating convection at large Prandtl numbers, focusing on centrifugal buoyancy effects that are usually neglected when using the Boussinesq 

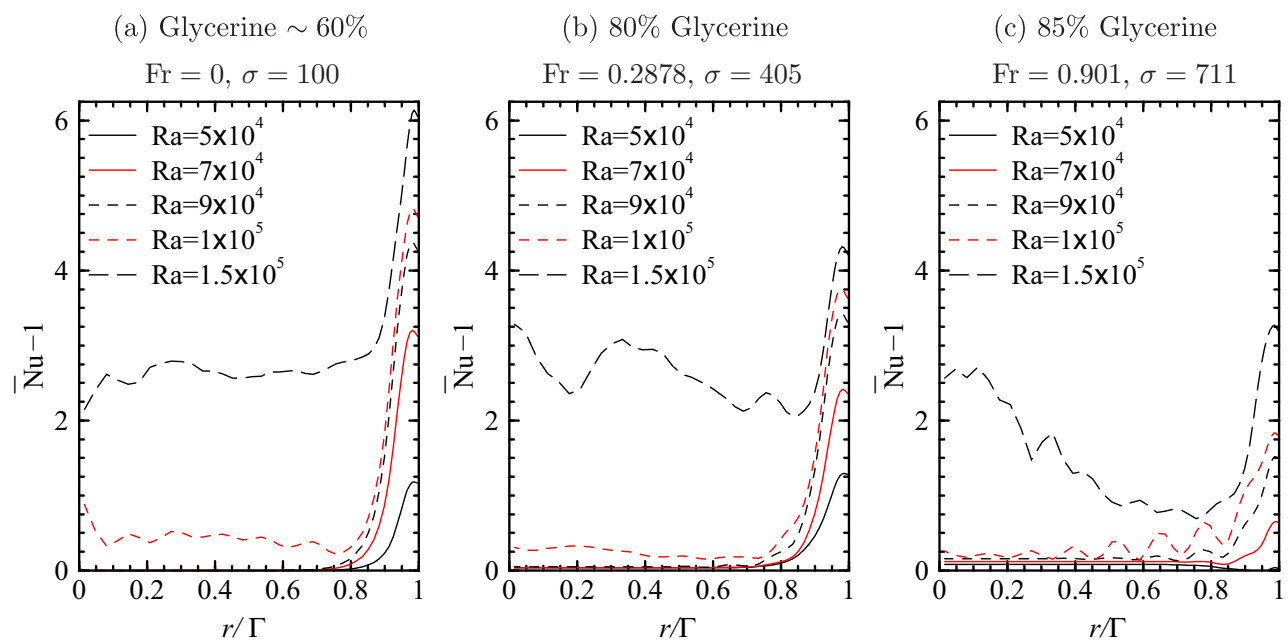

FIG. 7. (Color online) Radial variations of the azimuthally averaged Nusselt number for $\Omega=625, \Gamma=4$, and various Ra, Fr, and $\sigma$ as indicated.

approximation. The presence of the centrifugal term means that horizontal periodicity is lost; this is a major set back for analytic and asymptotic studies, as well as numerically, as Fourier basis functions in the horizontal directions cannot be used. However, the centrifugal buoyancy term is readily accommodated in appropriate numerical treatments, for example, using a Chebyshev basis in the radial direction.

Large Prandtl numbers are easily obtained using different concentrations of glycerine in aqueous mixtures, making the parameter ranges investigated in this study readily accessible in laboratory experiments. An additional advantage of using glycerine solutions is that the range of Prandtl and Froude numbers accessible is large: one and two orders of magnitudes, respectively, for the numerical simulations reported here.

We have analyzed the flow structure in the Rayleigh number range $5 \times 10^{4} \leqslant \mathrm{Ra} \leqslant 1.5 \times 10^{5}$, i.e., from the onset of wall modes up to convection in the bulk. We have found that the centrifugal buoyancy term has a large impact on the structure and dynamics of the sidewall boundary layer and also on the bulk convection.

In the absence of rotation, the flow characteristics change with increasing Prandtl number and at about $\sigma=100$ an asymptotic regime is reached where there are no further changes in the flow. However, in the presence of rotation, increasing $\sigma$ is linked to increments in Fr and new dynamics result. At low $\mathrm{Ra}$, increasing the glycerine concentration results in the wall modes losing angular velocity in the laboratory frame of reference (i.e., the retrograde rotation rate increases) due to the large-scale circulation induced by the centrifugal buoyancy, which eventually drives the wall modes towards target patterns with increasing Fr. For larger Ra, the wall modes are quenched, and if Fr is large enough the wall modes completely disappear. At large Ra, bulk convection appears in most of the cell, and if $\mathrm{Fr}=0$ is artificially set in the governing equations, the dynamics is related to the Küppers-Lortz dynamics. At higher Fr, the dynamics change as random narrow cold plumes emerging from the top lid emerge. These changes in the flow structure have a direct impact on the heat transfer. For small Fr, most of the heat transport is due to the wall mode plumes. As Fr increases and the wall modes are quenched, the heat transport is reduced, until Fr is large enough so that the centrifugal buoyancy-driven large-scale circulation leading to the axisymmetric target pattern flow is strong and accounts for the heat transport.

A major conclusion of the present study is that Fr effects cannot be ignored for rotating convection at large Prandtl numbers. The simulations at the largest Ra numbers explored show that the structure and dynamics of the plumes emerging from the top and bottom end wall boundary layers are significantly altered when Fr effects are included. The dynamics are very different from the Küppers-Lortz dynamics that are observed when $\mathrm{Fr} \approx 0$. This aspect warrants further detailed study both from the experimental and the numerical point of view.

\section{ACKNOWLEDGMENTS}

This work was supported in part by the US National Science Foundation grants DMS-0922864 and CBET-1336410, the Spanish Ministry of Education and Science grants FIS200908821, MTM2011-26696, and MINECO: ICMAT Severo Ochoa project SEV-2011-0087. The authors thank Isabel Mercader for discussions on the numerical aspects of this work.

\section{APPENDIX: TEMPERATURE-DEPENDENT VISCOSITY}

The nondimensional governing equations including temperature-dependent viscosity are

$$
\begin{aligned}
\left(\partial_{t}+\boldsymbol{u} \cdot \nabla\right) \boldsymbol{u}= & -\nabla p+\sigma \operatorname{Ra} T \hat{z}+2 \sigma \Omega \boldsymbol{u} \times \hat{z} \\
& -\frac{\sigma \operatorname{FrRa}}{\Gamma} T \boldsymbol{r}+\sigma \nabla \\
& \cdot\left[\frac{v(T)}{v_{0}}\left(\nabla \boldsymbol{u}+(\nabla \boldsymbol{u})^{T}\right)\right], \\
\left(\partial_{t}+\boldsymbol{u} \cdot \nabla\right) T= & \nabla^{2} T, \quad \nabla \cdot \boldsymbol{u}=0 .
\end{aligned}
$$

The equation $v_{C}(T)$ that approximates the dependence of viscosity with temperature for glycerine-water mixtures from Ref. [34] is used. Since in our setting the temperature range is small, we use a linear approximation for the temperature dependence of viscosity, given by the tangent to the curve 
reported in Ref. [34] at the reference temperature $T_{0}$. In dimensionless form, it is given by

$$
v(T)=v_{0}(1-\gamma T)
$$

where $\gamma$ is a linear rate given by

$$
\gamma=\epsilon \Delta T, \quad \epsilon=-\left.\frac{1}{v_{0}} \frac{d \nu_{C}}{d T}\right|_{T=T_{0}} .
$$

The viscous term in (A1), $\sigma \nabla \cdot\left\{\frac{v(T)}{\nu_{0}}\left[\nabla \boldsymbol{u}+(\nabla \boldsymbol{u})^{T}\right]\right\}$ that depends on the velocity, is expanded in a part that is independent of the temperature and is treated implicitly, and another part that explicitly depends on $T$ and is proportional to $\gamma$, that is treated explicitly in the same way as the Coriolis term, $\sigma \Omega \boldsymbol{u} \times \hat{z}$. This scheme works well for a weak dependency of viscosity on temperature and the set of Prandtl numbers considered in our study. However, it is not stable for stronger viscosity dependencies on temperature or infinite Prandtl number (see Ref. [35] for further details).
We have studied temperature-dependent viscosity effects by setting $\gamma$ equal to the values corresponding to the various glycerine concentrations considered. Fixing $\sigma=100$ and $\mathrm{Ra}=9 \times 10^{4}$, we tested this effect with and without centrifugal buoyancy, i.e., with $\mathrm{Fr}=0$ and Fr corresponding to various glycerine mixtures. We found that the relative differences in the horizontally averaged Nusselt number at either the bottom or top end wall obtained with constant viscosity $(\gamma=0)$ and with temperature-dependent viscosity are less than $0.2 \%$. Moreover, in all of the cases tested, the relative differences between the temperature fields at the point $(r, \theta, z)=(3.2,0,0)$ are below 5\%. Even at the largest Fr, the temperature-dependent viscosity case does not produce significant changes in the solution with respect to the case of constant viscosity. The same was observed at larger $\mathrm{Ra}=10^{5}$. These results are as to be expected given that for the parameter regimes used, $\alpha \Delta T<10^{-3}$, as noted earlier. Hence, since the effects of temperature-dependent viscosity are negligible and yet quite expensive to incorporate computationally, we have keep the viscosity constant for the result in the main body of this paper.
[1] M. Lappa, Rotating Thermal Flows in Natural and Industrial Processes (Wiley, New York, 2012).

[2] E. A. Spiegel, Annu. Rev. Astron. Astrophys. 9, 323 (1971).

[3] C. F. Chen and S. Thangam, J. Fluid Mech. 161, 161 (1985).

[4] E. M. King and J. M. Aurnou, Proc. Natl. Acad. Sci. USA 110, 6688 (2013).

[5] R. J. A. M. Stevens, H. J. H. Clercx, and D. Lohse, Phys. Rev. E 86, 056311 (2012).

[6] P. Constantin, C. Hallstrom, and V. Poutkaradze, J. Math. Phys. 42, 773 (2001).

[7] C. R. Doering and P. Constantin, J. Math. Phys. 42, 784 (2001).

[8] X. Yan, J. Math. Phys. 45, 2718 (2004).

[9] G. M. Homsy and J. L. Hudson, J. Fluid Mech. 35, 33 (1969).

[10] J. E. Hart, J. Fluid Mech. 403, 133 (2000).

[11] N. Becker, J. D. Scheel, M. C. Cross, and G. Ahlers, Phys. Rev. E 73, 066309 (2006).

[12] F. Marques, I. Mercader, O. Batiste, and J. M. Lopez, J. Fluid Mech. 580, 303 (2007).

[13] A. Rubio, J. M. Lopez, and F. Marques, J. Fluid Mech. 644, 337 (2010).

[14] J. M. Lopez and F. Marques, J. Fluid Mech. 628, 269 (2009).

[15] R. J. A. M. Stevens, H. J. H. Clercx, and D. Lohse, Eur. J. Mech. B Fluids 40, 41 (2013).

[16] F. H. Busse, J. Fluid Mech. 30, 625 (1967).

[17] D. D. Gray and A. Giorgini, Intnl J. Heat Mass Transfer 19, 545 (1976).

[18] J. M. Lopez, F. Marques, I. Mercader, and O. Batiste, J. Fluid Mech. 590, 187 (2007).
[19] F. Marques and J. M. Lopez, Phys. Fluids 20, 024109 (2008).

[20] S. P. Gregory, in Glycerine: A Key Cosmetic Ingredient, edited by E. Jungermann and N. O. V. Sonntag (Marcel Dekker, New York, 1991), pp. 113-156.

[21] S. Hughes and A. Randriamampianina, Int. J. Num. Meth. Fluids 28, 501 (1998)

[22] B. Fornberg, A Practical Guide to Pseudospectral Methods (Cambridge University Press, Cambridge, 1998).

[23] S. A. Orszag and A. T. Patera, J. Fluid Mech. 128, 347 (1983).

[24] I. Mercader, M. Net, and A. Falqués, Comp. Meth. Appl. Mech. Eng. 91, 1245 (1991).

[25] I. Mercader, O. Batiste, and A. Alonso, Comput. Fluids 39, 215 (2010).

[26] H. F. Goldstein, E. Knobloch, I. Mercader, and M. Net, J. Fluid Mech. 248, 583 (1993).

[27] J. M. Lopez, A. Rubio, and F. Marques, J. Fluid Mech. 569, 331 (2006).

[28] S. Chandrasekhar, Hydrodynamic and Hydromagnetic Stability (Oxford University Press, Oxford, 1961).

[29] J. H. P. Dawes, J. Fluid Mech. 428, 61 (2001).

[30] R. C. Kloosterziel and G. F. Carnevale, J. Fluid Mech. 480, 25 (2003).

[31] K. Julien and E. Knobloch, J. Math. Phys. 48, 065405 (2007).

[32] A. Prosperetti, Phys. Fluids 24, 114101 (2012).

[33] G. Küppers and D. Lortz, J. Fluid Mech. 35, 609 (1969).

[34] N. S. Cheng, Ind. Eng. Chem. Res. 47, 3285 (2008).

[35] J. Curbelo and A. M. Mancho, Commun. Nonlinear Sci. Numer. Simulat. 19, 538 (2014). 